returning from Northern Italy. Early modelling completed by the Department of Public Health in March 2020 estimated the potential for five hundred Covid-19 related hospital admissions. St. Bernard's Hospital is the only hospital in Gibraltar and has a capacity of around one hundred and fifty beds.

The key issues identified early on were identifying a venue of sufficient capacity to create a Covid-19 field hospital, oxygen requirements and the actual building of the facility. A recently completed sports facility was identified as being available which had sufficient capacity to provide spaces for three hundred beds.

The major challenge was ensuring adequate oxygen delivery to the field hospital. As it was in a remote location, oxygen had to be sourced independently of the main hospital. An operational decision was made to only admit patients who required $5 \mathrm{~L} / \mathrm{min}$ oxygen or less. A contingency plan was made to ensure oxygen cylinders could be charged within Gibraltar at two units: a newly commissioned oxygen plant and the local hyperbaric unit. The field hospital was operational by the time of the expected surge in Covid-19 cases.

An important lesson is that each field hospital will have differing specialist needs but have a backbone of common requirements. New hospitals will need staff, equipment and a physical space. However, during the Covid-19 pandemic an important issue that was flagged up early was the requirement for substantial amounts of oxygen. This helped to guide our team to build a specific hospital for a specific situation.

\section{Leadership and Management}

\section{LEADERSHIP DURING THE COVID-19 CRISIS: HOW DID WE DO AND HOW CAN WE DO BETTER?}

${ }^{1}$ Maria Henriksson, ${ }^{1}$ Jenna Jacqueline Godfrey, ${ }^{2}$ Jawaad Saleem Malik, ${ }^{2}$ Rachel Bartlett. ${ }^{1}$ Charing Cross Hospital, London, UK; 'St Mary's Hospital, London UK

\subsection{6/leader-2020-FMLM.135}

Background The COVID-19 pandemic posed unique challenges to healthcare workers, who were required to adapt to a changing environment. This crisis put the spotlight on leadership qualities to provide safe, high-quality care. Furthermore, the NHS adapted from a divisional to 'command and control' based leadership that risked staff disengagement and exclusion. Aim To understand how leadership and management across three teaching hospitals in London were handled during the pandemic, and how lessons learnt can be applied to improve future healthcare leadership.

Methods A survey was sent to all anaesthetic trainees across three teaching hospitals in London. This contained 10 questions concerning the quality of leadership and care observed during the pandemic.

Results $68 \%$ of trainees felt more valued during the pandemic. $63 \%$ felt more listened to and better able to express concerns. $50 \%$ felt less included in decisions made. Only 23\% thought NHS England and 14\% thought Public Health England exhibited good leadership and communication on how to prepare for and manage the crisis. Locally, 91\% reported good leadership within their teams, and 91\% agreed that patient care was sufficiently prioritised. Leadership qualities identified during the pandemic included compassion (reported by $86 \%$ ), difficult decision making (reported by $82 \%$ ) and staff engagement (reported by 68\%).

Conclusions The results suggest participants felt more valued and listened to than previously, and suggest compassionate leadership was exhibited on a local level. The pressure on leaders during the pandemic highlighted the need for developing leadership and management skills. However the majority of trainees $(68 \%)$ are not considering a career in leadership and management. We suggest implementing the NHS Leadership Academy's Healthcare Leadership Model App into anaesthetic training as a platform to engage trainees in developing their leadership skills, and provide the NHS with future healthcare leaders.

\section{Urology}

\section{REVIEWING THE CHANGE IN MANAGEMENT OF EMERGENCY RENAL COLIC SERVICES AND SURGICAL INTERVENTION IMPACTED BY COVID-19}

Caroline Woo, Mohammed Kamil Quraishi, Joseph Gabriel, Simon Mackie, Graham Watson. East Sussex NHS Health Trust

10.1136/leader-2020-FMLM.136

Background and Aim COVID-19 has resulted in changes to how the healthcare system in the United Kingdom delivered its emergency care, including renal colic and its management. Our stone team amended its services in order to reduce stone related morbidity and preventable hospital admissions. We aim to review similar time periods in 2019 and 2020 to see how this has changed.

Methods A retrospective review of all presentations of acute renal colic with CT confirmed stones were carried out for the months of April to May, 2019 and 2020. Demographics, clinical features and stone characteristics and treatment were noted.

Results Seventy nine percent of the 38 patients who present in 2019 had ureteric stones, compared to $85 \%$ of the 40 patients in 2020. Distal stones made up 53\% in 2019, compared to $56 \%$ in 2020 . Less patients were noted to be febrile in 2020 (13\%vs35\%).

There was an increase in conservative management in 2020 (25\% vs 21\%). Emergency treatment (stent/nephrostomy) with secondary intervention (Ureterorenoscopy(URS)/Percutaneous Nephrolithotomy (PCNL)) was markedly reduced in 2020 $(12.5 \%$ vs $42 \%)$.More patients were also treated with primary interventions (URS/PCNL) within 48 hours (43\% in 2020 vs $26 \%$ ) and 7 days (13\% vs $8 \%$ ).

Conclusion More patients were treated with conservative treatments and early primary interventions. This was done in order to combat unnecessary hospital admissions and stays, as well as surgeries and anaesthesia, whilst minimising stone and stent complications. An improved adherence to GIRFT guideless was also noted, providing better patient care and outcome, whilst reducing unnecessary procedures. 